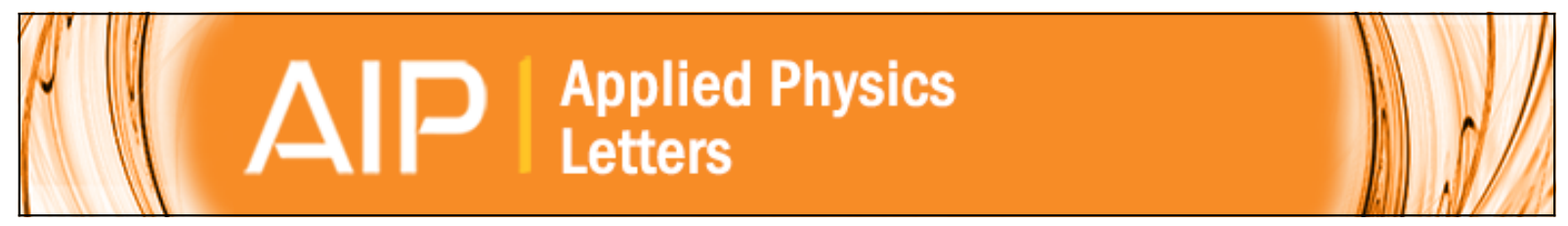

Memory and nonlocal effects in heat transport: From diffusive to ballistic regimes F. X. Alvarez and D. Jou

Citation: Applied Physics Letters 90, 083109 (2007); doi: 10.1063/1.2645110

View online: http://dx.doi.org/10.1063/1.2645110

View Table of Contents: http://scitation.aip.org/content/aip/journal/apl/90/8?ver=pdfcov

Published by the AIP Publishing 


\title{
Memory and nonlocal effects in heat transport: From diffusive to ballistic regimes
}

\author{
F. X. Alvarez ${ }^{\text {a) }}$ \\ Departament de Física, Universitat Autònoma de Barcelona, 08193 Bellaterra, Catalonia, Spain \\ D. Jou ${ }^{\text {b) }}$ \\ Departament de Física, Universitat Autònoma de Barcelona, 08193 Bellaterra, Catalonia, Spain \\ and Institut d'Estudis Catalans, Carme 47, Barcelona 08001, Catalonia, Spain
}

(Received 1 December 2006; accepted 16 January 2007; published online 22 February 2007)

The authors discuss a generalized transport model including memory and nonlocal effects, which aims to describe the transition of heat transport from the diffusive regime to the ballistic regime. By using an effective thermal conductivity depending on the Knudsen number, they describe in a single equation the behavior of conductivity in terms of the system size and a reduction in the limit flux through nanoscale devices. (C) 2007 American Institute of Physics. [DOI: 10.1063/1.2645110]

In the last years, several experiments or simulations on heat transport along nanoscale devices or one-dimensional model systems ${ }^{1-9}$ have obtained results that differ significantly from those of the classical Fourier law. In order to improve the results of the theory, some authors have proposed modifications to the diffusive heat conduction equation to achieve a wide description going from macrosystems to nanosystems. ${ }^{2,5}$ In these models, the heat flux is assumed to be the sum of two contributions: a ballistic one and a diffusive one. Such formalisms combine macroscopicFourier (or Cattaneo) equation for the diffusive part and microscopic-Boltzmann equation for the ballistic part, and some of them are inspired in the description of thermal radiation, as the equation of phonon radiative transfer (EPRT). ${ }^{4}$

Other authors have proposed a single contribution to the heat flux but characterized by a time-dependent thermal conductivity, ${ }^{10}$ or by a size-dependent thermal conductivity, which reduces to the classical thermal conductivity when the size of the system becomes much longer than the mean free path. ${ }^{6,11,12}$ Here we explore this latter proposal and compare it with other ones and with experimental and numerical results.

In classical thermodynamics, heat flux $\mathbf{q}$ is given by Fourier Law $\mathbf{q}=-\lambda \nabla T$, with $\lambda$ the thermal conductivity and $T$ the absolute temperature. To describe the temperature evolution, this equation is combined with the energy conservation equation and one is led to the equation

$$
\frac{\partial}{\partial t} T=\frac{\lambda}{c_{v}} \nabla^{2} T,
$$

with $c_{v}$ the specific heat per unit volume. The results of this equation fit correctly in systems where the mean free path of the particles is much shorter than the size of the system. As we apply it to smaller systems, the deviations with respect to the observations become more obvious. For instance, Larson et al. ${ }^{13}$ found that when a temperature difference is applied in

\footnotetext{
a)Electronic mail: xavier.alvarez@uab.es

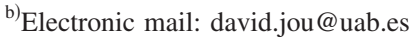

nanometer-scale silicon samples, the heat flux obtained is significantly lower than that predicted by Fourier law. The authors suggested that a reduction in the conductivity of the material may be the cause of this behavior.

In order to describe the behavior of nanoscale devices, several authors ${ }^{3,6,14}$ have tried to model the heat transport at this scale with equations including nonlocal effects, whose lack in Fourier's equation is the conceptual source of discrepancy with observations. Indeed, at scales comparable to the mean free path the particles move ballistically. One of such models is provided by extended irreversible thermodynamics (EIT), ${ }^{12}$ in which the Fourier heat conduction is modified in order that nonlocal and memory effects are taken in consideration. In the most sophisticated version of this theory, all the higher-order fluxes are considered as independent variables and the consecutive transport equations form a hierarchy of coupled evolution equations for the higher-order fluxes $\mathbf{J}^{(n)}, \mathbf{J}^{(1)}$ being the heat flux $\mathbf{q}, \mathbf{J}^{(2)}$ (a tensor of order of 2 ) the flux of the heat flux, and so on. From the point of view of kinetic theory this would correspond to take as variables higher-order moments of the distribution function. ${ }^{12,15}$ In a linear approximation, the equations of such hierarchy have the form ${ }^{12}$

$$
\begin{aligned}
& \nabla T^{-1}-\alpha_{1} \dot{\mathbf{J}}^{(1)}+\beta_{1} \nabla \cdot \mathbf{J}^{(2)}=\mu_{1} \mathbf{J}^{(1)}, \\
& \beta_{n-1} \nabla \dot{\mathbf{J}}^{(n-1)}-\alpha_{n} \dot{\mathbf{J}}^{(n)}+\beta_{n} \nabla \cdot \mathbf{J}^{(n+1)}=\mu_{n} \mathbf{J}^{(n)},
\end{aligned}
$$

with $\mu_{i}, \alpha_{i}$, and $\beta_{i}$ the phenomenological coefficients related to transport coefficients, relaxation times of the fluxes, and their respective correlation lengths, respectively. If we consider an infinite number of fluxes and work with the Fourier transform of Eqs. (2) and (3), we obtain the following expression for the heat flux:

$$
\mathbf{q}(\omega, k)=-i k \lambda(\omega, k) \hat{T}(\omega, k)
$$

where $\lambda(\omega, k)$ is the continued-fraction expression for a $k$ and $\omega$-dependent effective thermal conductivity, 


$$
\lambda(\omega, k)=\frac{\lambda_{0}(T)}{1+i \omega \tau_{1}+k^{2} l_{1}^{2} /\left\{1+i \omega \tau_{2}+k^{2} l_{2}^{2} /\left[1+i \omega \tau_{3}+k^{2} l_{3}^{2} /\left(1+i \omega \tau_{4}+\ldots\right)\right]\right\}},
$$

with $\lambda_{0}(T)$ the classical bulk thermal conductivity. The last two relations allow us to express the heat equation in the form

$$
-i \omega \hat{T}(\omega, k)=k^{2} \lambda(\omega, k) \hat{T}(\omega, k) .
$$

If we take, for simplicity, all the mean free paths and relaxation times equal $\left(\omega_{n}=\omega, l_{n}=l / 2\right)$, independently of the order of approximation, this fraction has the asymptotic limit,

$$
\lambda(\omega, k)=\frac{-(1+i \omega \tau)+\sqrt{(1+i \omega \tau)^{2}+(k l)^{2}}}{\frac{1}{2}(k l)^{2}} .
$$

The equality of all relaxation times and mean free paths for the higher-order fluxes is natural in a relaxation-time approximation for the evolution equation for the distribution function. Deeper analyses based on the full Boltzmann equation may lead to different values for the several relaxation times. ${ }^{11,15}$ Here, we explore the simplest possibility.

A detailed analysis based on Boltzmann equation ${ }^{15}$ shows that the lengths $l_{n}$ must be interpreted as $l / 2$, with $l$ the mean free path of heat carriers. If we are only interested in stationary behavior we can make $\omega=0$ and we obtain a size-dependent thermal conductivity by considering $L=2 \pi / k$. We are led to ${ }^{11}$

$$
\lambda(L)=\frac{\lambda_{0} L^{2}}{2 \pi^{2} l^{2}}\left[\sqrt{1+4\left(\frac{\pi l}{L}\right)^{2}}-1\right] .
$$

The ratio $l / L=K n$ is usually called the Knudsen number. When the mean free path is much shorter than the device, we have $\lim _{K n \rightarrow 0} \lambda(L)=\lambda_{0}$. In the opposite situation, one has $\lim _{K n \rightarrow \infty} \lambda(L) \simeq\left(\lambda_{0} / \pi\right)(L / l)$.

The behavior of heat conductivity [Eq. (8)] in terms of the size of the device is plotted in Fig. 1. The dependence of the conductivity with the width of the system fits to the val-

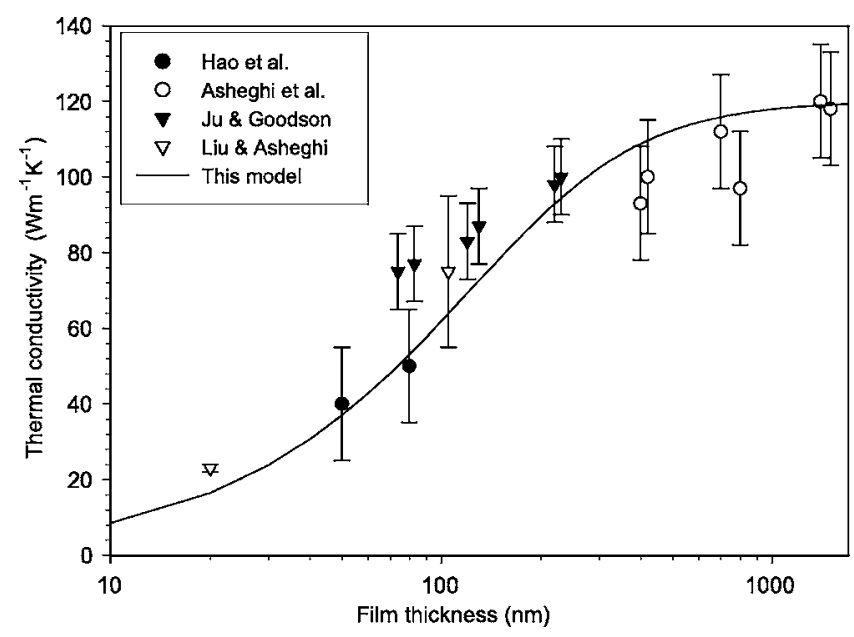

FIG. 1. Effective-thermal conductivity $(\lambda)$ in $\mathrm{W} /(\mathrm{m} \mathrm{K})$ given by Eq. (8) in terms of the width of the layer $(L)$ in $\mathrm{nm}$. The values used in the graph are $120 \mathrm{~W} /(\mathrm{cm} \mathrm{K})$ for the asymptotic conductivity $\lambda_{0}$ and $40 \mathrm{~nm}$ for $l$. The points correspond to the experimental data in lavers with different thicknesses (see Refs. 16 and 17). ues obtained by Liu and Asheghi ${ }^{16}$ and Hao et al. ${ }^{17}$ in inplane heat conductivity experiments through very thin layers. The theoretical result for Eq. (8) is compared with the plotted points using the bulk conductivity of silicon of $120 \mathrm{~W} \mathrm{~m}^{-1} \mathrm{~K}^{-1}$ and the mean free path of phonons of $40 \mathrm{~nm}$ as fitting parameter. The form of Eq. (8) also yields a reasonable fit to the size-dependent effective conductivity in nanotubes, according to the experimental values reported in Ref. 18.

When the size of the system is much longer than the mean free path, the conductivity does not depend on the size and its value is the classical thermal conductivity. In contrast, when the size is of the same order or lower than the mean free path, the conductivity starts to fall and it tends to zero as the size tends to zero. For high values of the Knudsen number, it behaves linearly with the size (this means that the heat flux depends only on the temperature difference and not on the temperature gradient). Such a reduction in the value of the effective conductivity is indeed observed in Ref. 13.

Now, we use the effective thermal conductivity Eq. (8) to obtain the evolution of the temperature profile for different values of the Knudsen number (in particular, for $K n=10$ ), and compare with other descriptions, a topic which has received much attention. ${ }^{2,4,10,19}$

In this case we must consider the full description Eqs. (4) and (5), which leads to a hyperbolic equation with an effective relaxation time $\tau_{\mathrm{eff}} \simeq \tau\left(1-l^{2} \omega / 4 \lambda \tau\right)=\tau / 4{ }^{12}$ Here we have taken into account that $\lambda=(1 / 3) c_{v} l v$, with $v$ the average velocity of the carriers. Now, we may write the evolution equation for temperature profile, expressing $t$ in terms of the collision time $\tau=l / v$ and $x$ as $x^{\prime}=x / L$, with $L$ the length of the system, and we get

$$
\frac{1}{4} \frac{\partial^{2} T}{\partial t^{2}}+\frac{\partial T}{\partial t}=\frac{1}{6 \pi^{2}}\left(\sqrt{1+4 \pi^{2} K n^{2}}-1\right) \nabla^{2} T .
$$

For low values of $K n$ this expression tends to

$$
\frac{1}{4} \frac{\partial^{2} T}{\partial t^{2}}+\frac{\partial T}{\partial t}=\frac{K n^{2}}{3} \nabla^{2} T,
$$

which is the hyperbolic version of Eq. (1). Usually, instead of the normalized effective time $\tau_{\text {eff }}$, one directly considers $\tau$ and the factor of $1 / 4$ does not appear in the first term. This factor has been experimentally observed in ultrasound velocity in gases. ${ }^{20}$

We take as initial conditions $T(x)=0$. At $t=0$ one suddenly imposes $T(0)=1$ at one end and one keeps $T(1)=0$ at the opposite end. In Fig. 2 the time evolution for the heat flux on the $x=0$ boundary is shown. We observe that the long-time limit for the flux in Maxwell-Cattaneo model is the same as in Fourier model, while the limit flux in EIT model is significantly lower. This flux reduction is analogous to that obtained by Chen (Fig. 3 of Ref. 2) with his two-component ballistic-diffusive model and agrees with the one observed experimentally. ${ }^{13}$ The advantage of our one-component model with generalized conductivity is that no rescaling of 


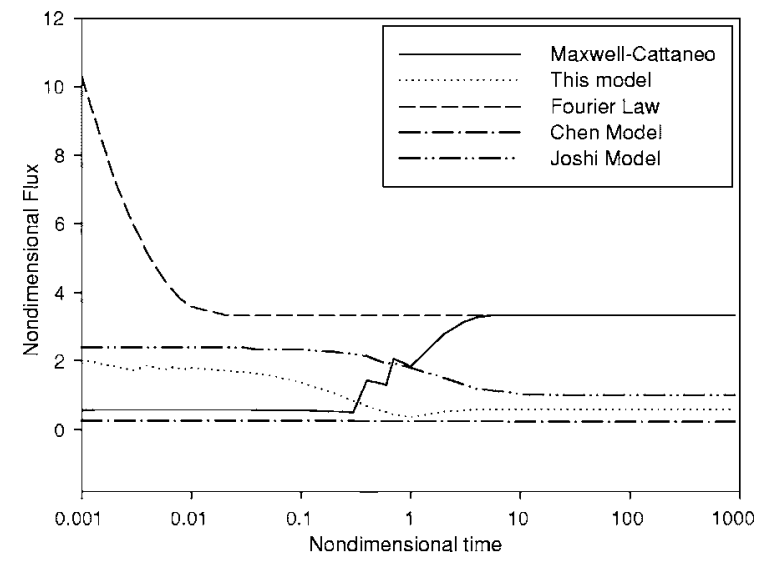

FIG. 2. Evolution of the heat flux through the $x=0$ wall in a device with Knudsen number of 10 . The dashed line represents the Fourier law, the solid line is the Maxwell-Cattaneo equation, the dotted line is the EIT equation, and the dash-dotted lines are Chen model (Ref. 19) and model by Joshi and Majumdar (Ref. 4).

temperature or heat flux has been needed in Fig. 2. In Chen's model, a rescaling is needed because it uses different temperatures for each type of particle. In our model this is not needed as only one equilibrium temperature is used. As the Knudsen number decreases the results become similar to those of Maxwell-Cattaneo and are not represented here.

Joshi and Majumdar ${ }^{4}$ used EPRT to obtain the evolution of the temperature over a rod by solving some profiles depending on the Knudsen number; they solved the propagation equation of ballistic phonons assuming that the emission of the latter on the boundaries follows the same law as the blackbody radiation. A problem of this model is the excessive time consumption when it is numerically resolved. The plot in Fig. 2 shows that EIT model yields similar behavior for the heat flux with a significantly simpler equation that is solvable analytically.

$\mathrm{Chen}^{2}$ used a simpler heat equation to obtain the same results as the EPRT. With this aim he classified the phonons in two classes: ballistic and diffusive. The latter follows Cattaneo equation, while ballistic phonons evolve according to the Boltzmann equation. As the relative abundance of ballistic particles is increased, the deviation from the classic equation becomes higher. In order to model the system with two types of particles with two transport equations, two temperatures must be defined, one for each type of particles and a different boundary value is imposed on each type of particles. The diffusive phonons act as if they were in a potential well, diffusing through all the system but bouncing at the walls. Their initial temperature is $T_{0}$ everywhere. The only way they have to increase their temperature is to scatter with ballistic phonons. This mechanism injects energy to the diffusive phonons, removing it from the ballistic ones. In Ref. 2, the results for this model are compared with those of Maxwell-Cattaneo hyperbolic equation, and are found to be closer to the observed behavior.

Naqvi and Waldenstrøm ${ }^{10}$ follow the single-component point of view and solve the heat equation with a timedependent conductivity, namely,

$$
\frac{\partial T}{\partial t}=\left(1-e^{-t / \tau}\right) \frac{K n^{2}}{3} \nabla^{2} T
$$

where $\tau=l / v$ is the mean free time. The boundary values imposed are fixed temperatures on both ends of the rod. The profiles that they obtain fit well to the ones obtained by Joshi and Majumdar. ${ }^{4}$

The solution of this model has not the wave front present in Chen's model and no rescaling of the temperature must be done. The difference between Eqs. (9) and (11) is that the conductivity is explicitly time dependent in Eq. (11), whereas in Eq. (9) it depends on the length of the system $L$. Both models obtain a lowering of the temperature profile because their ultimate effect is an effective reduction of the thermal conductivity.

In summary, we have studied a generalized thermal conductivity [Eq. (8)] depending on the Knudsen number, which gives a reasonable approximation to the behavior of thermal conductivity in nanotubes and thin layers in steady state conditions. We have solved numerically the evolution equation [Eq. (9)] for the temperature profile, for Knudsen number of 10 where discrepancies from the classical limit are expected. The results obtained for the long-time value of the heat flux if Fig. 2 with the generalized thermal conductivity [Eq. (8)] are similar to those obtained by Chen., ${ }^{2,19}$ Furthermore, our approach provides an alternative to Naqvi and Waldenstrøm who proposed a time-dependent thermal conductivity, but with the advantage that in the steady state our model is able to describe the thermal conductivity of nanotubes and thin layers, in contrast with Ref. 10.

The present model describes, in one single equation, the observed behavior of the conductivity in nanoscale devices, ${ }^{16-18}$ a reduction in the limit flux ${ }^{13}$ and a simplified equation for the temperature profile that does not require the separation in ballistic and diffusive parts.

The authors acknowledge the financial support of the Spanish Ministry of Education under Grant No. FIS200612296-C02-01 and of Direcció General de Recerca of the Generalitat of Catalonia under Grant No. 2005SGR00087.

${ }^{1}$ J. Wang and J.-S. Wang, Appl. Phys. Lett. 88, 111909 (2006).

${ }^{2}$ G. Chen, Phys. Rev. Lett. 86, 2297 (2001).

${ }^{3}$ S. S. Ghai, W. T. Kim, R. A. Escobar, C. H. Amon, and M. S. Jhon, J. Appl. Phys. 97, 10P703 (2005).

${ }^{4}$ A. A. Joshi and A. Majumdar, J. Appl. Phys. 74, 31 (1993).

${ }^{5}$ S. Lepri, R. Livi, and A. Politi, Phys. Rep. 377, 1 (2003).

${ }^{6}$ J. Che, T. Çagin, and W. A. Goddard III, Nanotechnology 11, 65 (2000).

${ }^{7}$ P. Kim, L. Shi, A. Majumdar, and P. L. McEuen, Phys. Rev. Lett. 87, 215502 (2001)

${ }^{8}$ M. Fujii, X. Zhang, H. Xie, H. Ago, K. Takahashi, T. Ikuta, H. Abe, and T. Shimizu, Phys. Rev. Lett. 95, 065502 (2005).

${ }^{9}$ D. C. Cahill, W. K. Ford, K. E. Goodson, G. D. Mahan, A. Majumdar, H. J. Maris, R. Merlin, and S. R. Phillpot, J. Appl. Phys. 93, 793 (2003).

${ }^{10}$ K. R. Naqvi and S. Waldenstrom, Phys. Rev. Lett. 95, 065901 (2005).

${ }^{11}$ D. Jou, J. Casas-Vazquez, G. Lebon, and M. Grmela, Appl. Math. Lett. 18, 963 (2005).

${ }^{12}$ D. Jou, J. Casas-Vazquez, and G. Lebon, Extended Irreversible Thermodynamics, 3rd ed. (Springer, Berlin, 2001), Chap. 5, p. 140.

${ }^{13}$ B. C. Larson, J. Z. Tischler, and D. M. Mills, J. Mater. Res. 1, 144 (1986).

${ }^{14}$ Q. Z. Xue, Nanotechnology 17, 1655 (2006).

${ }^{15}$ S. Hess, Z. Naturforsch. A 32a, 678 (1977).

${ }^{16}$ W. Liu and M. Asheghi, Appl. Phys. Lett. 84, 3819 (2004).

${ }^{17}$ Z. Hao, L. Zhichao, T. Lilin, T. Zhimin, L. Litian, and L. Zhijian, Chin. J. Semicond. 27, 1961 (2006).

${ }^{18}$ D. Li, Y. Wu, P. Kim, L. Shi, P. Yang, and A. Majumdar, Appl. Phys. Lett. 83, 2934 (2003)

${ }^{19} \mathrm{G}$. Chen, J. Heat Transfer 124, 320 (2002).

${ }^{20}$ A. M. Anile and S. Pluchino, J. Mec. Theor. Appl. 3, 167 (1984) 